\title{
Corrigendum
}

\section{Corrigendum to "Inhibiting Interleukin 17 Can Ameliorate the Demyelination Caused by A. cantonensis via iNOS Inhibition"}

\author{
Ying Feng, ${ }^{1}$ Cunjing Zheng, ${ }^{2}$ Feng Feng, ${ }^{3}$ Shuo Wan, ${ }^{4}$ Xin Zeng, ${ }^{4}$ Fukang Xie $\mathbb{D},{ }^{2}$ \\ and Zhongdao $\mathrm{Wu} \mathbb{D}^{4}$ \\ ${ }^{1}$ Medical School of South China University of Technology, Guangzhou, Guangdong 510006, China \\ ${ }^{2}$ Histology and Embryology Department of Zhongshan School of Medicine, Sun Yat-sen University, Guangzhou 510080, China \\ ${ }^{3}$ The Department of Pharmacology and Toxicology, School of Pharmaceutical Sciences, Sun Yat-sen University, \\ Guangzhou 510080, China \\ ${ }^{4}$ Parasitology Department of Zhongshan School of Medicine, Sun Yat-sen University, Guangzhou 510080, China
}

Correspondence should be addressed to Fukang Xie; frankxie2000@yahoo.com and Zhongdao Wu; wuzhd@mail.sysu.edu.cn

Received 26 March 2018; Accepted 3 April 2018; Published 2 May 2018

Copyright (c) 2018 Ying Feng et al. This is an open access article distributed under the Creative Commons Attribution License, which permits unrestricted use, distribution, and reproduction in any medium, provided the original work is properly cited.

In the article titled "Inhibiting Interleukin 17 Can Ameliorate the Demyelination Caused by $A$. cantonensis via iNOS Inhibition" [1], the names of the first, second, fourth, sixth, and seventh authors were reversed. The revised authors' list is shown above.

\section{References}

[1] F. Ying, Z. Cunjing, F. Feng et al., "Inhibiting interleukin 17 can ameliorate the demyelination caused by A. cantonensis via iNOS inhibition," Mediators of Inflammation, vol. 2017, Article ID 3513651, 7 pages, 2017. 


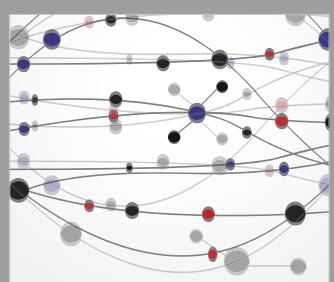

The Scientific World Journal
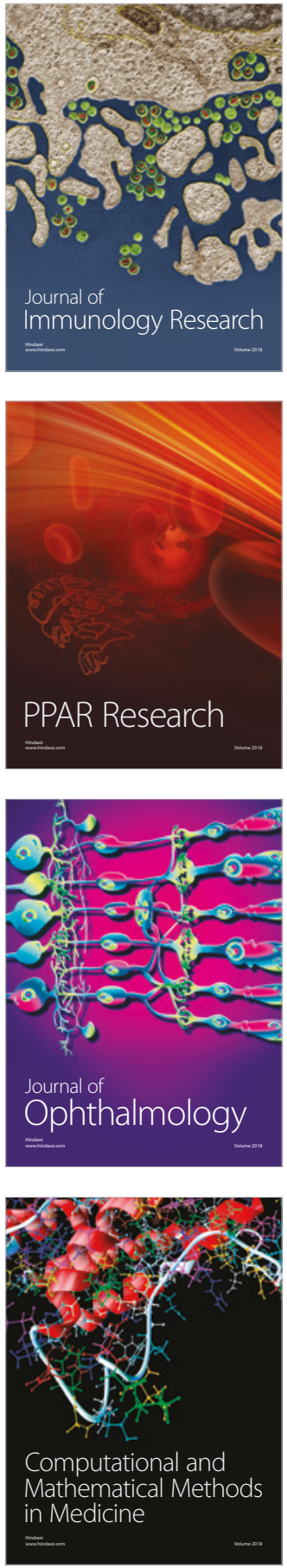

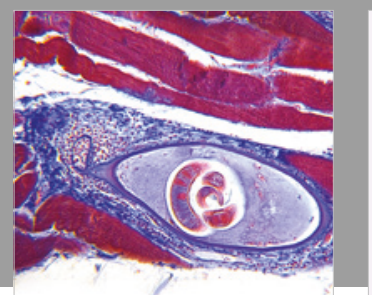

Gastroenterology Research and Practice

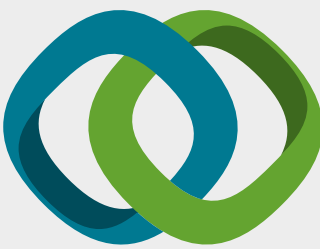

\section{Hindawi}

Submit your manuscripts at

www.hindawi.com
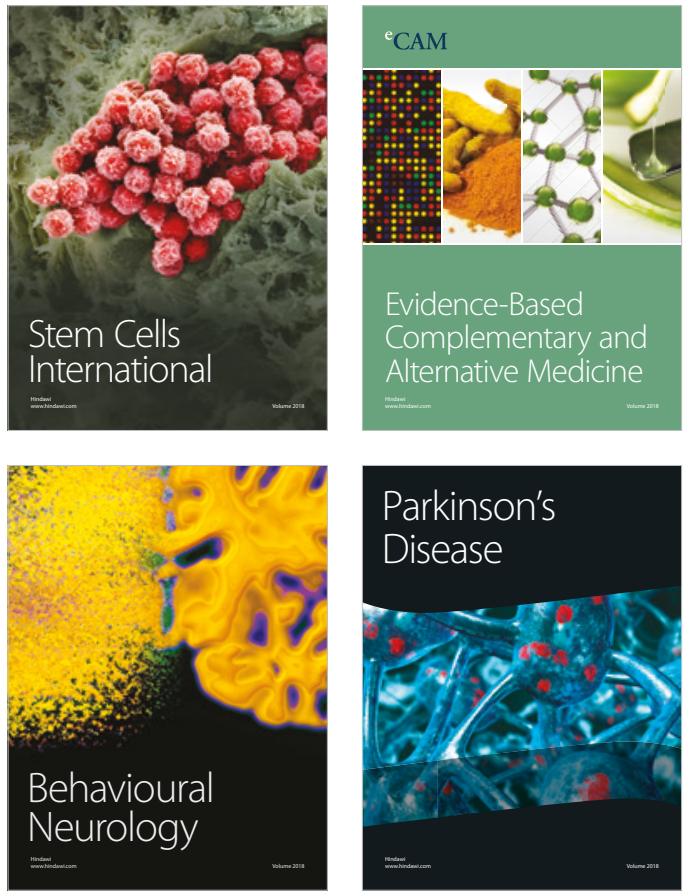

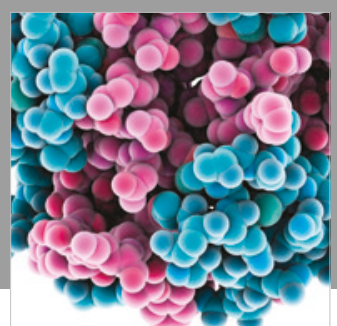

ournal of

Diabetes Research

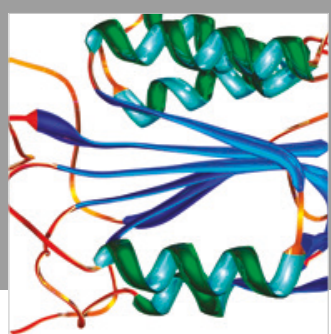

Disease Markers
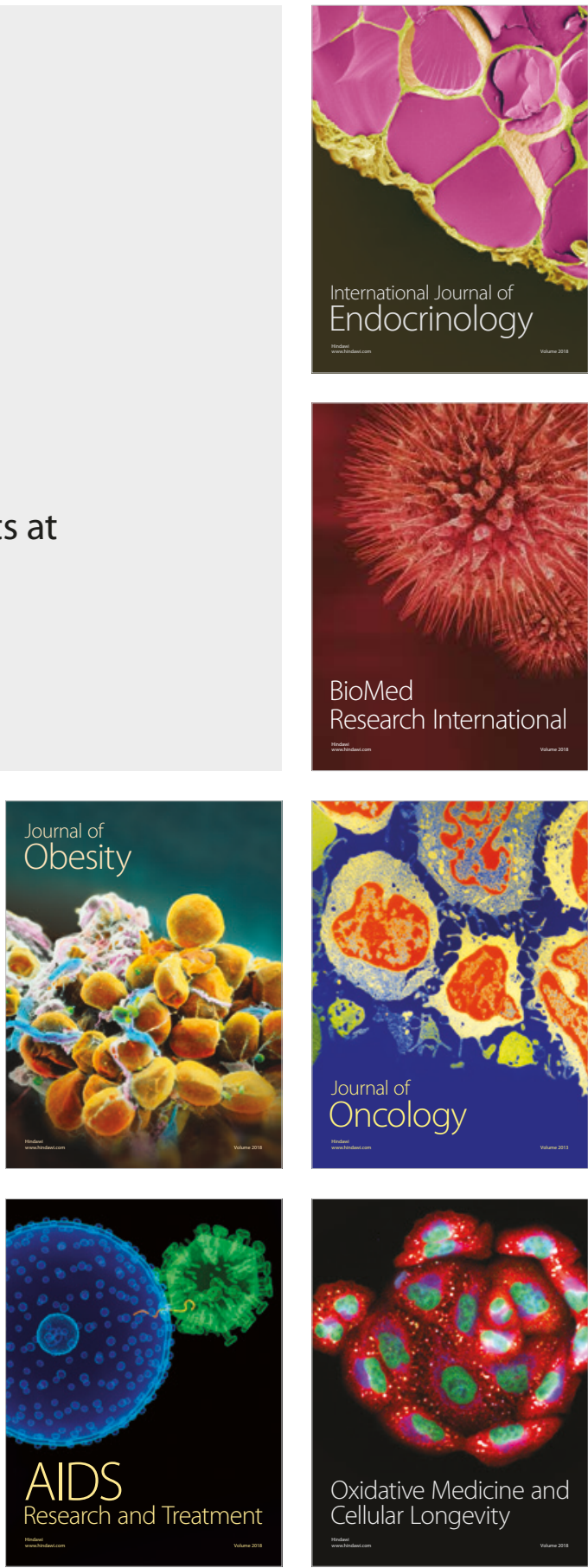